\title{
Total hip arthroplasty for Crowe type IV developmental dysplasia of the hip combined with intertrochanteric fracture: a case report and literature review
}

\author{
Wei Chen ${ }^{1}$, Yong $\mathrm{Ma}^{1}$, Hui Ma ${ }^{1}$ and Mao $\mathrm{Nie}^{2^{*}}$ (1)
}

\begin{abstract}
Background: Total hip arthroplasty for Crowe type IV developmental dysplasia of the hip (DDH) is a complex procedure. Crowe type IV DDH combined with intertrochanteric frature is very rare.

Case presentation: A 75-year-old patient suffering from left hip pain after a fall was sent to our hospital. Plain radiographs and computed tomography scans were used to diagnose this patient with DDH combined with an intertrochanteric fracture. We conducted a total hip arthroplasty using an S-rom prosthesis following subtrochanteric shortening osteotomy in this patient, after which steel wires were used to fix the intertrochanteric fracture. The patient did not suffer any significant intraoperative or postoperative complications, and treatment was sufficient to overcome lower leg abnormalities. The patient was encouraged to resume walking with support at 3 days post-surgery, and at 6-month postoperatively he had regained the majority of his original range of motion. At 10-month postoperatively, the intertrochanteric fracture and subtrochanteric osteotomy of left femur had healed effectively,and the patient's VAS and mHSS scores had improved significantly.
\end{abstract}

Conclusions: Total hip arthroplasty is an effective approach to treat patients suffering from Crowe type IV DDH combined with an intertrochanteric fracture, and can achieve satisfactory clinical outcomes.

Keywords: Developmental dysplasia of the hip, Intertrochanteric fracture, Total hip arthroplasty

\section{Background}

Crowe type IV developmental dysplasia of the hip (DDH) is a severe deformity that results in hip dysfunction and an abnormal gait [1]. This condition is typically treated via total hip arthroplasty (THA), which is a technically demanding procedure. Intertrochanteric hip fractures commonly occur among older adults. However, there have not been any reports regarding the appropriate

\footnotetext{
*Correspondence: 302218@cqmu.edu.cn

${ }^{2}$ Department of Orthopaedic Surgery, The Second Affiliated Hospital of Chongqing Medical University, 76 Linjiang Road, Yuzhong District, Chongqing 400010, People's Republic of China

Full list of author information is available at the end of the article
}

treatment of patients simultaneously suffering from both DDH and an intertrochanteric hip fracture. In the present report, we describe a rare case of a patient that presented with type IV DDH combined with an intertrochanteric fracture. This patient achieved good clinical outcomes following treatment via THA with subtrochanteric shortening osteotomy and greater trochanter fixation.

\section{Case presentation}

A 75-year-old male patient was sent to our hospital suffering from left hip pain sustainedly after an accidental fall. Radiographic imaging revealed that this patient was affected by Crowe type IV DDH combined with an Evans 
type III intertrochanteric fracture of left femur (Fig. 1a). The patient reported a history of left hip trauma at the age of 10 , at which time he did not accept proper treatment. The resultant left hip deformities had since caused the patient to walk with a limp. Prior to his fall, the patient was able to bear weight and work with moderate left hip pain. Physical examination revealed the left leg to be $4 \mathrm{~cm}$ shorter than the right leg, with local tenderness being detected in the left hip. In addition, percussive pain in the left femur in the axial direction and pain with internal and external rotation were noted. As passive activity caused serious pain, we were unable to measure the degrees of left hip motion. The patient had been diagnosed with hypertension for six years, and regularly took blood pressure control medications. Computed tomography scans revealed the high posterior dislocation of the left femoral head up to the level of the greater sciatic foramen(Fig. 1b). The original acetabulum was smaller and shallower (Fig. 1b). In order to better preoperatively evaluate the acetabulum and femur deformities in this patient, a 3D printed model was constructed (Fig. 1c).

The patient was offered two surgical options: THA or internal fixation to treat only the intertrochanteric fracture. After careful consideration and discussions with family members and the surgeon, the patient selected to undergo THA, and also provided informed consent for the publication of his case, which has not been reported previously to our knowledge.

Under general anesthesia, the patient was placed in the lateral decubitus position. The operation was performed via a posterior approach with an incision length of almost $20 \mathrm{~cm}$. The external rotators were first detached, and then the femur neck was removed. After resection of the elongated hypertrophic joint capsule, the original acetabulum was clearly exposed and gradually reamed to $50 \mathrm{~mm}$. Next, the $50 \mathrm{~mm}$ acetabular cup and a $28 \mathrm{~mm}$ polyethylene liner were placed in an appropriate anatomic arrangement with three screws. To clearly expose the proximal femoral canal, the femoral great trochanter fragment was overturned along the fracture line. Approximately $2 \mathrm{~cm}$ below the lesser trochanter, a $4 \mathrm{~cm}$-long femoral shortening transverse osteotomy was performed by resecting the femur in accordance with preoperative planning. A modular S-rom femur stem was then installed from the level of intertrochanteric fracture end into the canal, and the femur was then de-rotated and the resected cylindric bone segment was cut longitudinally, after which these two pieces were bound onto the
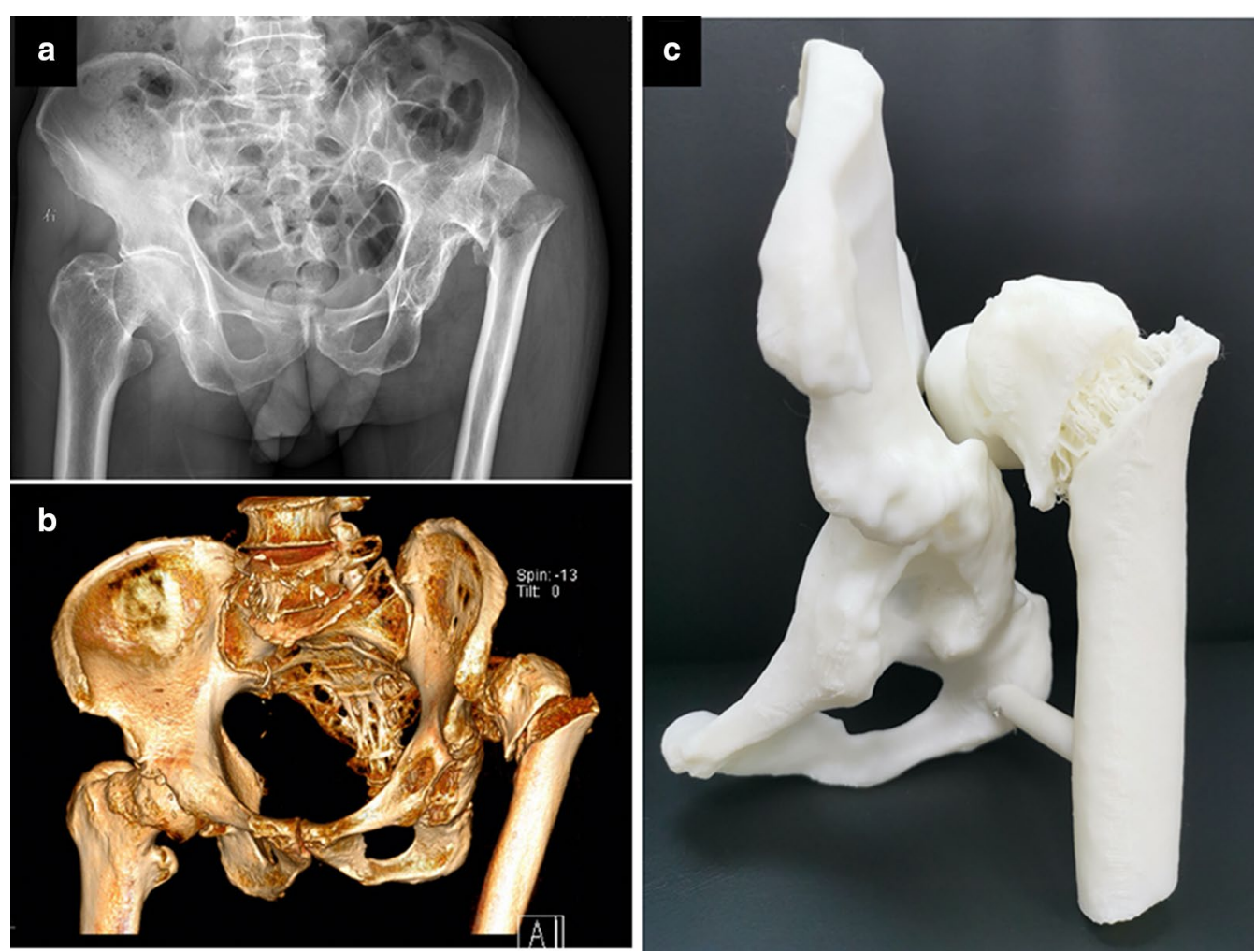

Fig. 1 a Preoperative anteroposterior radiographs of the pelvis and hip. b Preoperative 3D computed tomography reconstruction model of the pelvis and hip. c Preoperative 3D-printed model of the pelvis and hip using the computed tomography data 
Table 1 Patient clinical scores

\begin{tabular}{lllll}
\hline & Before injury & 3 months postoperatively & 6 months postoperatively & 10 months postoperatively \\
\hline VAS & 5 & 1 & 0 & 0 \\
mHHS & 53 & 73 & 84 & 87 \\
\hline
\end{tabular}

osteotomy site with wires. Equipped with $32 \mathrm{~mm}$ short metal head, the stem was easily reduced, after which the intertrochanteric fracture was reduced and stably fixed with steel wires.

Postoperatively, the patient was administered intravenous antibiotics and prophylactic anti-thrombotic treatment. Three days postoperatively, the patient was encouraged to stand and to walk with the aid of a walker. Postoperative plain radiographic images revealed that the original rotational center of the hip was restored and that the inclination and anteversion of the cup had been restored (Fig. 2a, b). The patient completed baseline visual analog scale (VAS) score and modified Harris hip score (mHHS) assessments via retrospective questionnaire, and also completed these assessments at 3 and 6 and 10 months postoperatively. Both VAS and mHHS scores were significantly improved at these postoperative follow-up time points (Table 1). Radiographic and computed tomography scans conducted upon most recent follow-up revealed that the prosthesis was properly positioned and the intertrochanteric fracture and subtrochanteric osteotomy had healed effectively (Figs. 2c, d, 3). Leg length discrepancy (LLD) measurements through the full length weight bearing radiograph of lower extremities showed the left limb to be $1 \mathrm{~cm}$ shorter than the right limb(Fig. 2e). Even though there was a little heterotopic ossification occurred on greater trochanter of left femur,the patient almost felt
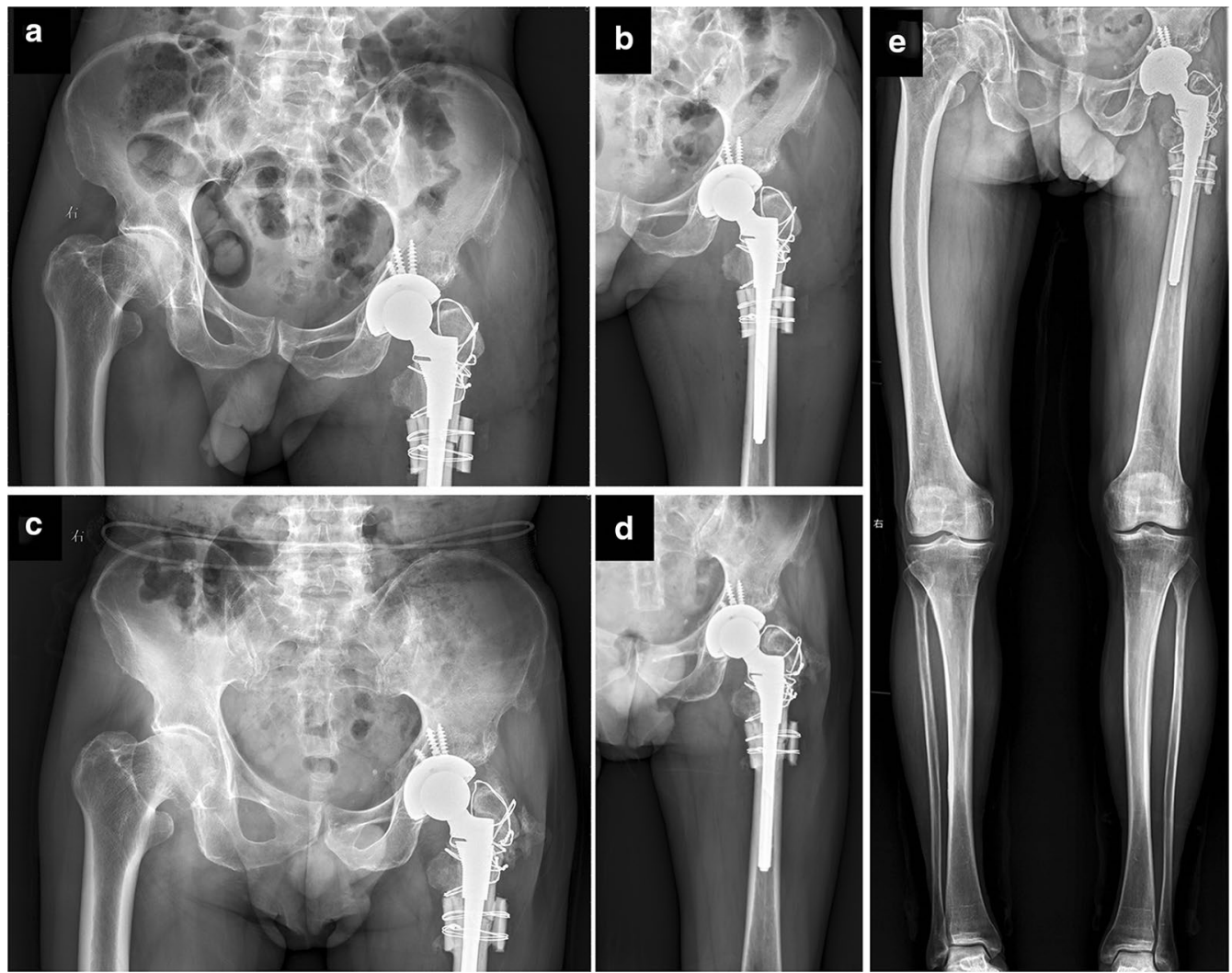

Fig. 2 Anteroposterior radiographs of the pelvis and left hip joint at 3-day postoperatively (a and $\mathbf{b}$ ) and 10-month follow-up postoperatively (c and $\mathbf{d}$ ). e Full length weight bearing radiograph of lower extremities at 10-month follow-up postoperatively 


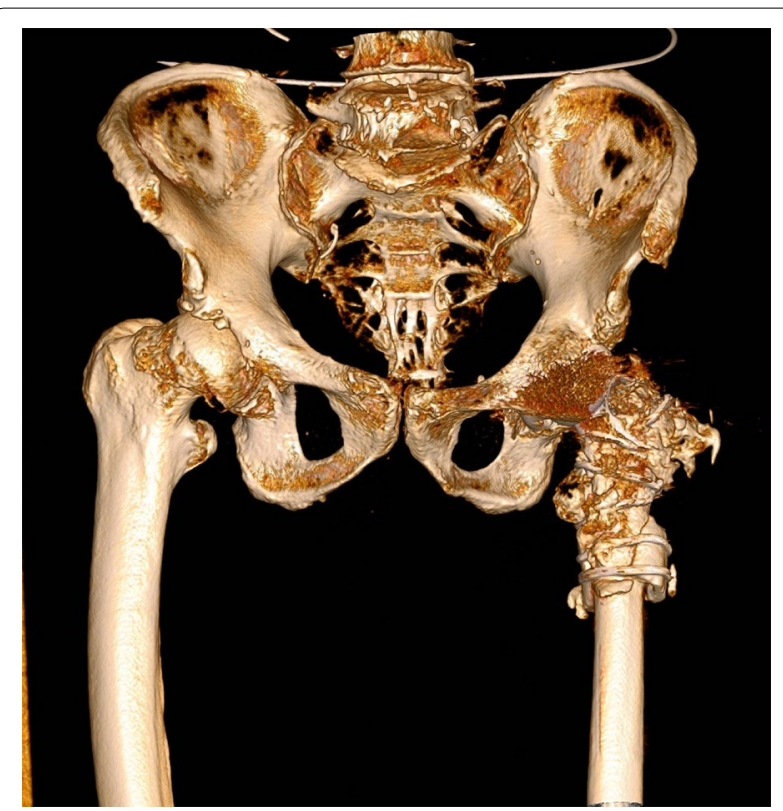

Fig. 3 3D computed tomography reconstruction model of the pelvis and hip at 10-month follow-up postoperatively

no pain and could walk up and down stairs without any assistance.

\section{Discussion and conclusions}

DDH is a common disorder in infants and children. Early detection and non-operative management has been shown to be effective for the management of pediatric DDH [2]. When such non-operative treatments fail, surgical approaches are recommended. With increasing age, the associated deformities can become more complex, necessitating increasingly aggressive treatments including open reduction and femoral or pelvic osteotomy [3]. For symptomatic DDH in young adults, joint-preserving surgeries such as hip arthroscopy, periacetabular osteotomy (PAO), femoral head-neck junction osteochondroplasty combined with PAO, intertrochanteric osteotomy, or capsular arthroplasty are recommended to patients with minimal articular cartilage degeneration, as they could reduce or delay the degeneration of the hip joint [4]. A recent systematic review revealed that hip arthroscopy for borderline DDH with capsular plication improved short-term patient-reported outcome measures [5].

Crowe type IV DDH is a severe deformity that results from the long-term dislocation of the femoral head, leading to femoral head deformities, a high hip center, a short femoral neck with excessive anteversion, a narrow femoral canal, a hypoplastic true acetabulum with reduced depth and deficient superior bone stock, a posteriorly located greater trochanter, and soft tissue contractures [6]. Given that this condition can result in gait abnormalities and hip dysfunction, treatments primarily aim to achieve anatomic hip reconstruction in order to correct lower limb discrepancies, thereby increasing the odds of achieving satisfactory functional outcomes. THA is the most common treatment for DDH in adults [7]. However, the rates of complications associated with this treatment approach are significantly higher than those associated with other forms of primary THA [8-10].

With respect to the surgical technique, the acetabular cup should be placed into the true acetabulum, and a modular femoral prosthesis is recommended for the deformed medullary cavity. A number of different osteotomy approaches including greater trochanter osteotomy, lesser trochanter osteotomy, and subtrochanteric osteotomy are available as a means of achieving reduction and eliminating gait abnormalities while minimizing the risk of nerve complications. Of these osteotomy techniques, transverse osteotomy is the simplest and reliably achieves positive outcomes $[6,8,9,11,12]$. Common complications following THA for the treatment of Crowe Type IV DDH include LLD, intraoperative fracture, osteotomy nonunion, and nerve injuries, making this procedure more challenging to conduct [13]. Preoperative individualized measurement and osteotomy calculations are important steps to reducing LLD. Physical therapy and shoe lifts can be utilized to address LLD > 5-10 mm after THA. Internal fixation is a common means of addressing intraoperative fracture. As the sciatic nerve should be carefully dissected and palpated during operation, osteotomy is an effective method to avoid any sciatic nerve injury. To reduce sciatic nerve tension, the ipsilateral knee should be maintained in flexion when the limb is lengthened [13].

Intertrochanteric hip fractures are among the most common fractures affecting the elderly, and are primarily treated via internal fixation with intramedullary fixation. However, owing to the relatively significant failure rates of internal fixation and the advantages of arthroplasty, certain surgeons conduct primary hip arthroplasty for the treatment of unstable intertrochanteric hip fractures and achieve positive outcomes [14-16]. Nie et al. [17] conducted a meta-analysis comparing intramedullary fixation and arthroplasty as approaches to the treatment of intertrochanteric hip fractures in the elderly, and they found that arthroplasty was associated with lower rates of reoperation and implant-associated complications. However, intramedullary fixation was associated with reduced blood loss, lower transfusion requirements, shorter operative duration, higher Harris hip scores, and lower 1-year mortality rates. 
Given the special circumstances associated with the present case, the internal fixation for treatment of intertrochanteric fractures could not relieve the symptoms caused by Crowe IV DDH.Total hip arthroplasty could be a better choice for this patient and it was not difficult to deal with the intertrochanteric fracture at the same time. Subtrochanteric shortening osteotomy was a safe way to correct the deformity of femur and facilitate reduction. The modular S-rom stem could provide secure fixation and stability for load bearing early. The patient did not suffer from any severe intraoperative or postoperative complications, and follow-up conducted at 10 months post-surgery indicated that his pain had been relieved and that his hip function was acceptable. The patient was satisfied with the treatment and is still undergoing regular follow-up. Overall, this case report is intended to provide orthopedic surgeons with an option to treat Crowe IV DDH combined with intertrochanteric fracture.

\section{Abbreviations}

DDH: Developmental dysplasia of the hip; THA: Total hip arthroplasty; LLD: Leg length discrepancy; VAS: Visual analog scale; mHHS: Modified Harris hip score; PAO: Periacetabular osteotomy.

\section{Acknowledgements}

Not applicable.

\section{Authors' contributions}

WC, YM, HM and MN performed the surgery. HM collected and interpreted the patient's clinical data.WC and MN drafted the manuscript. MN contributed to the revision. All authors read and approved the final manuscript.

\section{Funding}

This work was funded by the Kuanren Talents Program of the Second Affiliated Hospital of Chongqing Medical University to Mao Nie.

\section{Availability of data and materials}

All data are included in the section of Case Presentation and are available from the corresponding author on reasonable request.

\section{Ethics approval and consent to participate}

Not applicable.

\section{Consent for publication}

Written informed consent was obtained from the patient for publication of this case report and any accompanying images. A copy of the written consent is available for review.

\section{Competing interests}

The authors declare that they have no competing interests.

\footnotetext{
Author details

1 Department of Orthopaedic Surgery, People's Hospital of Fengjie County, Chongqing 404600, People's Republic of China. ${ }^{2}$ Department of Orthopaedic Surgery, The Second Affiliated Hospital of Chongqing Medical University, 76 Linjiang Road, Yuzhong District, Chongqing 400010, People's Republic of China.
}

\section{References}

1. Sanchez-Sotelo J, Berry DJ, Trousdale RT, Cabanela ME. Surgical treatment of developmental dysplasia of the hip in adults: II. Arthroplasty options. J Am Acad Orthop Surg. 2002;10(5):334-44.

2. Mulpuri K, Song KM. AAOS Clinical Practice Guideline: detection and nonoperative management of pediatric developmental dysplasia of the hip in infants up to six months of age. J Am Acad Orthop Surg. 2015;23(3):206-7.

3. Murphy RF, Kim YJ. Surgical management of pediatric developmental dysplasia of the hip. J Am Acad Orthop Surg. 2016;24(9):615-24.

4. Chen M, Shang XF. Surgical treatment for young adult hip dysplasia: jointpreserving options. Int Orthop. 2016;40(5):891-900.

5. Kuroda Y, Saito M, Sunil Kumar KH, Malviya A, Khanduja V. Hip arthroscopy and borderline developmental dysplasia of the hip: a systematic review. Arthroscopy. 2020;36(9):2550-2567 e2551.

6. Necas L, Hrubina M, Melisik M, Cibula Z, Chmurny M, Daniel M, Steno B. Cementless hip arthroplasty and transverse shortening femoral osteotomy with the S-ROM stem for Crowe type IV developmental dysplasia. Eur J Orthop Surg Traumatol. 2019;29(5):1025-33.

7. Rogers BA, Garbedian S, Kuchinad RA, Backstein D, Safir O, Gross AE. Total hip arthroplasty for adult hip dysplasia. J Bone Jt Surg Am. 2012;94(19):1809-21.

8. Li Y, Zhang X, Wang Q, Peng X, Wang Q, Jiang Y, Chen Y. Equalisation of leg lengths in total hip arthroplasty for patients with Crowe type-IV developmental dysplasia of the hip: classification and management. Bone Jt J. 2017;99(7):872-9.

9. Wang D, Li LL, Wang HY, Pei FX, Zhou ZK. Long-term results of cementless total hip arthroplasty with subtrochanteric shortening osteotomy in Crowe type iv developmental dysplasia. J Arthroplasty. 2017;32(4):1211-9.

10. Zeng WN, Liu JL, Wang FY, Zhang $X$, Fan $H Q$, Chen GX, Guo L, Duan XJ, Zhou Q, Yang L. Total hip arthroplasty for patients with Crowe type IV developmental dysplasia of the hip: ten years results. Int J Surg. 2017:42:17-21.

11. Atilla B. Reconstruction of neglected developmental dysplasia by total hip arthroplasty with subtrochanteric shortening osteotomy. EFORT Open Rev. 2016;1(3):65-71.

12. Krych AJ, Howard JL, Trousdale RT, Cabanela ME, Berry DJ. Total hip arthroplasty with shortening subtrochanteric osteotomy in Crowe type-IV developmental dysplasia: surgical technique. J Bone Jt Surg Am. 2010;92(Suppl 1 Pt 2):176-87.

13. Shi XT, Li CF, Han Y, Song Y, Li SX, Liu JG. Total hip arthroplasty for Crowe type IV hip dysplasia: surgical techniques and postoperative complications. Orthop Surg. 2019;11(6):966-73.

14. Kim SY, Kim YG, Hwang JK. Cementless calcar-replacement hemiarthroplasty compared with intramedullary fixation of unstable intertrochanteric fractures. A prospective, randomized study. J Bone Jt Surg Am. 2005;87(10):2186-92.

15. Bonnevialle P, Saragaglia D, Ehlinger M, Tonetti J, Maisse N, Adam P, Le Gall C, French H, Knee S, Trauma Surgery A. Trochanteric locking nail versus arthroplasty in unstable intertrochanteric fracture in patients aged over 75 years. Orthop Traumatol Surg Res. 2011;97(6 Suppl):S95-100.

16. Fichman SG, Makinen TJ, Safir O, Vincent A, Lozano B, Kashigar A, Kuzyk PR. Arthroplasty for unstable pertrochanteric hip fractures may offer a lower re-operation rate as compared to cephalomedullary nailing. Int Orthop. 2016;40(1):15-20.

17. Nie B, Wu D, Yang Z, Liu Q. Comparison of intramedullary fixation and arthroplasty for the treatment of intertrochanteric hip fractures in the elderly: a meta-analysis. Medicine (Baltimore). 2017;96(27):e7446.

\section{Publisher's Note}

Springer Nature remains neutral with regard to jurisdictional claims in published maps and institutional affiliations. 